\title{
Efecto de la Integración de las Artes Visuales en el Desarrollo de la Lectura y la Escritura
}

\section{Effect of the Integration of Visual Arts in Reading and Writing Skills}

\author{
Wanda I. Padilla Martínez * \\ Jaime Ortiz-Vega
}

Pontificia Universidad Católica de Puerto Rico - Puerto Rico

\begin{abstract}
La educación actual enfrenta grandes desafíos. Anualmente, en Puerto Rico, gran parte de los estudiantes toman pruebas estandarizadas en las que deben demostrar dominio de las competencias en el área académica de español, entre otras. Este artículo presenta una investigación cuyo propósito fue determinar el efecto de la integración de las artes visuales, durante diez semanas, en el desarrollo de las destrezas de lectura y escritura en estudiantes de tercer grado de escuelas públicas del suroeste de Puerto Rico. Se utilizó un enfoque cuantitativo con un diseño cuasi-experimental para determinar la diferencia entre: las puntuaciones de la posprueba de lectura y de escritura, respectivamente, de los estudiantes a los que se le aplicó el tratamiento y a los que no se le aplicó; las puntuaciones de la preprueba y la posprueba de lectura y de escritura, respectivamente, obtenida por los estudiantes de cada grupo. La muestra de 21 estudiantes de tercer grado se seleccionó por conveniencia, en dos escuelas similares. De éstos, 14 formaron parte del grupo control (GC) y 17 del grupo experimental (GE). Los datos se recolectaron mediante la pre y posprueba de lectura y la pre y posprueba de escritura creada por la investigadora. La información recopilada se analizó mediante estadística inferencial paramétrica (t test, ANOVA, regresión lineal) y estadística no paramétrica (Mann Whitney, Wilcoxon). Los resultados evidenciaron diferencias significativas entre GC y GE, en los promedios de las puntuaciones de las prepruebas y de las pospruebas de lectura y escritura, respectivamente. Estos hallazgos sugieren que la integración de las artes visuales tuvo un efecto significativo en el desarrollo de las destrezas de lectura y escritura.
\end{abstract}

Palabras clave: Pruebas estandarizadas; Destrezas del lenguaje; Desarrollo de lectura; Destrezas de escritura; Educación en arte; Estrategias de aprendizaje.

Today's education faces major challenges. Annually, in Puerto Rico, most of the students take standardized tests in which they must demonstrate mastery in the competences in the academic area of Spanish, among others. This article presents an investigation whose purpose was determine the effect of the integration of visual arts, for ten weeks, in the development of Reading and writing skills in third grade students in public schools in southwest of Puerto Rico. A quantitative approach was used with a quasi-experimental design to determine the difference between: the post-test scores of reading and writing, respectively, of the students to whom the treatment was applied and to whom it was not applied; the pre-test and post-test scores of reading and writing, respectively, obtained by the students of each group. The sample of 21 third graders was selected for convenience in two similar schools. Of these, 14 were part of the control group (CG) and 17 of the experimental group (EG). The data were collected through the pre and post reading test and the pre and post writing test created by the researcher. The information collected was analyzed by parametric ( $\mathrm{t}$ test, ANOVA, lineal regression) and nonparametric (Mann Whitney, Wilcoxon) inferential statistics. The results evidenced significant differences between CG y EG, in the averages of the pre and post Reading and Writing test scores, respectively. These findings suggest that the integration of the visual arts had a significant effect on the development of Reading and writing skills.

Keywords: Standardized Tests; Language Skills; Reading Skills; Writing Development; Art Education; Learning Strategies.

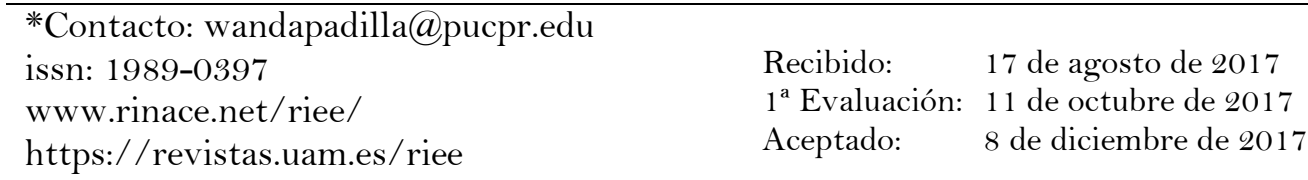




\section{Introducción}

En una era en que se ha logrado, a pasos agigantados, un avance en los diversos campos del saber se buscan maneras nuevas de fortalecer el aprendizaje de modo que éste trascienda a las disciplinas (Krishna, 2004; Santiago-Villafañe y Figarella-García, 2012). En este contexto, el Departamento de Educación de Puerto Rico (DEPR) se enfrenta al reto de transformar las escuelas con el propósito de mejorar el aprovechamiento académico de los estudiantes (DEPR, 2014a). Según lo establece el DEPR, la enseñanza de las artes del lenguaje en español es una de las áreas de prioridad. De acuerdo con DEPR (2015a) siendo el español la lengua materna, es el principal medio de comunicación que regula la interacción entre los seres humanos que hablan esta lengua y, por consiguiente, es el lenguaje con que los estudiantes aprenden los conocimientos de las demás áreas del saber. El dominio del idioma, según el DEPR (2014b), provee las habilidades para el devenir de las nuevas situaciones en un mundo dinámico al servir como apoyo una formación integral que tiene como resultado, educandos activos, críticos y participativos.

En Puerto Rico, los estudiantes de escuela pública pasan por un proceso de medición anualmente denominado Pruebas Puertorriqueñas de Aprovechamiento Académico (PPAA). A partir del año 2016, estas pruebas se denominan Medición y Evaluación para la Transformación Académica de Puerto Rico (META-PR). Estas pruebas, alineadas a los estándares académicos establecidos por el DEPR, están diseñadas para evaluar el aprovechamiento académico en diferentes materias, entre estas, español. La ley No Child Left Behind (NCLB, 2002) requiere que los estudiantes matriculados en los grados 3er, 4to, 5to, 6to, 7mo, 8 vo y $11 \mathrm{vo}$, participen de este programa de medición. El cuadro 1 presenta información relacionada con el desempeño de los estudiantes del DEPR en las PPAA durante los años 2012 a 2015.

Se observa en el cuadro 1, que los estudiantes en los años 2012 a 2015 no alcanzaron la meta anual estipulada por las leyes federales que rigen la educación en Puerto Rico (PR). De acuerdo con el DEPR (2015b) los estudiantes han tenido dificultad en demostrar dominio en el área de español. La situación se complica, ya que en el área académica de español las metas anuales son más elevadas cada año (DEPR, 2014a). En lel cuadro 2 se presentan los objetivos medibles anuales (Annual Measurable Objectives, o AMO) que establece el sistema de educación diferenciada en el Plan de Flexibilidad (DEPR, 2014a; USDE, 2015) para la materia de español, para los grados de tercero a octavo.

Cuadro 1. Desempeño de los estudiantes en PPAA (DEPR, 2015b)

\begin{tabular}{|c|c|c|c|c|c|c|c|c|}
\hline & 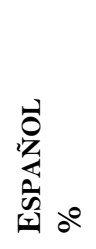 & 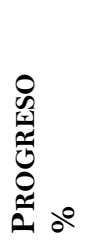 & 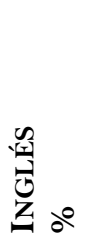 & 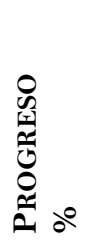 & 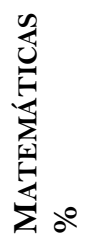 & 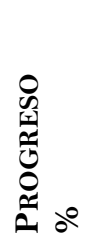 & $\stackrel{0}{6}$ & 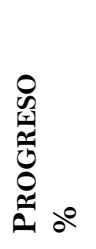 \\
\hline 2012-2013 & 47,88 & & 42,61 & & 28,78 & & 39,75 & \\
\hline $2013-2014$ & 45,71 & $-2,17$ & 41,35 & $-1,26$ & 30,03 & 1,24 & 39,02 & $-0,73$ \\
\hline $2014-2015$ & 43,55 & $-2,16$ & 41,91 & 0,56 & 29,64 & $-0,39$ & 38,36 & $-0,66$ \\
\hline
\end{tabular}


Cuadro 2. Objetivos medibles anuales (AMO) para español, 3er a 8vo grado (DEPR, 2014a)

\begin{tabular}{|c|c|c|c|c|c|c|c|}
\hline \multicolumn{8}{|c|}{ LÍNEA BASE } \\
\hline & $\begin{array}{c}2011- \\
2012 \\
\%\end{array}$ & $\begin{array}{c}\text { 2012- } \\
2013 \\
\%\end{array}$ & $\begin{array}{c}2013- \\
2014 \\
\%\end{array}$ & $\begin{array}{c}2014- \\
2015 \\
\%\end{array}$ & $\begin{array}{c}2015- \\
2016 \\
\%\end{array}$ & $\begin{array}{c}2016- \\
2017 \\
\%\end{array}$ & $\begin{array}{c}2017- \\
2018 \\
\%\end{array}$ \\
\hline $\begin{array}{l}\text { Todos los } \\
\text { estudiantes }\end{array}$ & 47,7 & 52,1 & 56,4 & 60,8 & 65,1 & 69,5 & 73,9 \\
\hline
\end{tabular}

Fuente: Elaboración propia.

El panorama actual de la educación pública, para los grados K-12 en Puerto Rico, es el resultado de no haber podido cumplir con la ley federal Elementary and Secondary Education Act (ESEA)(1965) según enmendada por la ley 107-110 (2002) conocida como No Child Left Behind (NCLB). La mencionada ley requería que los estudiantes lograran el 100 por ciento de proficiencia académica para el año 2014, sin embargo, el objetivo no se logró. No obstante, en el 2011, el Departamento de Educación de Estados Unidos (DEEU) ofreció a los estados y territorios la oportunidad de hacer un plan para acogerse a las dispensas otorgadas a requisitos particulares de la ley ESEA. El DEPR presentó un Plan de Flexibilidad al DEEU con el objetivo de lograr el dominio de los estándares y la oportunidad de mejorar el desempeño de los estudiantes, según sus necesidades diferenciadas (DEPR, 2014a). Asimismo, indica que la meta de ese sistema de rendición de cuentas es aumentar la transparencia en los procesos educativos y lograr que los grupos de interés se involucren en el proceso de transformar las escuelas, de manera que esto resulte en un sistema de escuelas públicas que satisfagan las necesidades de los estudiantes.

De acuerdo con el Informe del Perfil Escolar (DEPR, 2015b) los estudiantes de tercer grado de las escuelas públicas de PR en las cuales se administró las PPAA en el año escolar 2014-2015, el 57,4\% obtuvieron puntuaciones equivalentes a avanzados y proficientes en la parte de español. En el cuadro 3 se presenta el nivel de proficiencia, en el área de español, de los estudiantes de tercer grado comparado con los años anteriores y con los AMO establecidos para cada año.

Cuadro 3. Proficiencia en español en las PPAA, según DEPR (2015b)

\begin{tabular}{ccccc}
\hline $\begin{array}{c}\text { AÑOS } \\
\text { ESCOLARES }\end{array}$ & $\begin{array}{c}\text { 3ER GRADO } \\
\text { \% }\end{array}$ & $\begin{array}{c}\text { PROGRESO } \\
\text { \% }\end{array}$ & $\begin{array}{c}\text { AMO } \\
\text { 3ER-8VO GRADO } \\
\text { \% }\end{array}$ & $\begin{array}{c}\text { CUMPLIÓ } \\
\text { META }\end{array}$ \\
\hline $2012-2013$ & 55,6 & ----- & 52,1 & NO \\
$2013-2014$ & 55,1 & $-0,5$ & 56,4 & NO \\
$2014-2015$ & 57,4 & 2,3 & 60,8 & NO \\
\hline
\end{tabular}

Fuente: Elaboración propia.

Si se toma en consideración que la meta anual, según la ley 107-110 (2002), para esta materia era de $83,18 \%$ y que, en el nuevo sistema diferenciado, los objetivos anuales medibles establecidos, era de $60,8 \%$, según el cuadro 3 , se observa que los estudiantes no dominaron las destrezas del lenguaje, tales como lectura, escritura y comprensión lectora, en los porcentajes esperados.

En este contexto, cabe preguntarse, ¿cuáles acciones se deben tomar para revertir ese estado de situación?, ¿cuál es la probabilidad de que mediante la integración de otras áreas curriculares en la enseñanza de las artes del lenguaje en español se pueda lograr resultados 
conducentes a cumplir con las metas establecidas? Si fuera así, ¿¿cuál es el efecto de la integración de las artes visuales en otras áreas curriculares, en particular el español? De acuerdo con Fiske (1999) de varias investigaciones se desprende que mediante la educación artística se puede transformar el proceso educativo al provocar, entre otros, el aprendizaje por descubrimiento, el mejoramiento de las relaciones interpersonales y la iniciativa de aprender. Según expone la política pública de la organización y la oferta curricular del programa de Bellas Artes en los niveles elemental y secundario de las escuelas públicas (DEPR, 2013), ésta provee al estudiante experiencias de comunicación universal que son necesarias para desenvolverse en el mundo del siglo XXI. Además, Maeso-Rubio (2003) sostiene, refiriéndose a la educación artística que, entre las diversas áreas de enseñanza, es una de las que posee unas características especiales que la diferencian de otras disciplinas.

De acuerdo con lo antes expuesto, surgen otras interrogantes tales como: ¿Cuál es el desempeño de los estudiantes en las destrezas de lectura y escritura? ¿Cuál es el rol de la lectura y la escritura en el desempeño académico? ¿Cuál es la importancia de las artes visuales en la educación?

Si se examinan los orígenes de la educación en bellas artes, según Eisner (1972) se notará que éstas han sido catalogadas como un área de estudio que no va más allá de lo que es el producto estético. Asimismo, en los últimos tiempos, las culturas han considerado las artes como una materia de poca importancia en una sociedad llena de riqueza que, además, posee mucho tiempo disponible (Sousa, 2002).

En Puerto Rico, no fue hasta el 1955, que se creó el Programa de Artes Visuales (DEPR, 2003a). Desde ese momento, la educación artística ha sido parte del currículo en las escuelas públicas de la Isla. Este programa pasó por procesos de consolidación y separación hasta que, en el 2000 su estructura se fortaleció a través de estándares académicos en los diferentes niveles de enseñanza. El DEPR (2003b) documenta que es a partir de esta fecha en que los enfoques de enseñanza se actualizaron y se alinearon a las nuevas tendencias educativas que favorecerían la implantación de estrategias para la expresión artística.

\section{Fundamentación teórica}

De las investigaciones se desprende que el proceso natural de alfabetización se inicia antes de la instrucción formal en la escuela (Goodman y Altwerger, 1981; Harste, Burke y Woodward, 1982; Mass, 1982; Ruiz, 1998). Es, precisamente esta conceptualización temprana del lenguaje impreso como proceso de comunicación, un elemento clave para el desarrollo eficaz de las destrezas de lectura y la escritura desde las etapas iniciales en el aprendizaje formal hasta la realización del lector hábil para toda la vida (Kontos y Huba, 1983). En este sentido, Fons (2010) afirma que es necesario propiciar un ambiente rico en experiencias de lectura y escritura que permita que los niños entren en contacto con una variedad de situaciones que se resuelvan mediante actos en los que tendrán que leer y escribir.

Goodman (2003) señala que el lenguaje se aprende en el proceso de su uso, mediante la participación activa y repetida en actividades que impliquen hablar y escribir. De esta manera, según el autor, se llevará a cabo un proceso natural del aprendizaje del lenguaje, 
denominado lenguaje integral; lo cual significa que el aprendiz va apropiándose del lenguaje desde su nacimiento y a través de todas las experiencias que impliquen el desarrollo de la actividad comunicativa.

Leer, según Solé (2010) es un proceso dialéctico entre un texto y un lector, proceso en el que éste aporta su disposición emocional y afectiva, sus propósitos, su experiencia, su conocimiento del mundo y del tema. Es esa experiencia, de acuerdo con el autor, la que permite comprender y construir un significado respecto a lo leído. Esta comprensión, será más profunda en la medida en que el estudiante pueda asociar su experiencia como lector con los conceptos que surgen del mundo de lo cotidiano (Freire, 2004).

Según Schneuwly (2010) el lenguaje escrito, cuya construcción es una de las tareas prioritarias del DEPR, supone la reorganización de distintas capacidades que forman una nueva esencia interior. También afirma el autor, que como parte de este proceso, que hace visible el idioma en uso, se requiere la utilización de diversas funciones intelectuales desde las más elementales a las más complejas para crear una combinación específica a través de las que se construye una nueva unidad. Tal es así, que Vygotsky (1995) sostiene que la escritura es el álgebra del lenguaje.

La práctica de la lectura y la escritura son procesos que se nutren recíprocamente (Rolón, 2013). No obstante, cuando se separan con la intención de fortalecer uno más que otro, según señala Dollins (2001) se debilita esa relación. De acuerdo con este autor, que llevó a cabo una investigación cualitativa sobre la escritura, en la que se ofreció ayuda remediativa a estudiantes rezagados en lectura. La evidencia reveló una transferencia del contenido utilizado en el proyecto cuando la conexión entre lectura y escritura era explícita.

En una investigación similar, Coady (2007) demostró la relación existente entre la lectura y la escritura. En medio de un panorama en el que los fondos que recibían las escuelas daban un particular énfasis a la lectura mediante el proyecto Leer primero, el autor decidió retar al sistema para lo cual llevó a cabo la investigación Que ningún escritor se quede atrás. Utilizó como marco de referencia, las teorías constructivistas de Vygotsky (1995) y Dewey (1934). Además, el enfoque de Graves (2004) que postuló que "la escritura es la realización de la lectura” (p. 4). Graves reclutó a un maestro voluntario para enseñar a un grupo dando énfasis a la enseñanza de la escritura. Como resultado de la investigación, se identificó la lectura y escritura como procesos que están conectados en la alfabetización.

En otra investigación, Reyes-Tosta (2013) expuso que "el mismo gobierno puertorriqueño ha admitido que su sistema de educación ha fracasado en su intento de lograr que los estudiantes dominen las destrezas de comunicación verbal y escrita del español” (p. 44). Según el autor, los resultados de las PPAA, que ofrece el DEPR, muestran que los estudiantes carecen del dominio de las destrezas básicas del lenguaje. El autor recomienda que, para lograr el éxito de éstos en ambientes educativos avanzados, se debe fomentar la lectura y escritura, y a su vez, la confianza y la competencia necesaria para desarrollar las mismas.

\subsection{Las artes visuales y el lenguaje}

Deasy (2002) presentó un compendio, el cual contiene 62 investigaciones sobre las relaciones determinantes entre el aprendizaje en las bellas artes, el aprovechamiento académico y el desempeño social. Mediante un análisis de los resultados obtenidos en cada 
una de estas investigaciones, Catterall (2002) sostiene que se comprobó que aquellas escuelas que incorporaron la enseñanza y aprendizaje alrededor de las artes visuales en el currículo experimentaron amplios logros tanto en el aprovechamiento académico como en la actitud positiva que se generó en los estudiantes respecto a la motivación para aprender. De acuerdo con el autor, de estas investigaciones se infiere que cada disciplina de las artes visuales se asocia con logros importantes. Establece, por ejemplo, que el dibujo ayudó a desarrollar las destrezas de escritura, mientras que el entrenamiento visual ayudó a la interpretación de textos. Del mismo modo, el estímulo de la imaginación es fundamental en el desarrollo intelectual ya que se trata de ver las cosas de una forma diferente a como son en realidad, siendo esto "lo que hacen los científicos y los artistas; perciben lo que es, pero imaginan lo que podría ser y luego usan su conocimiento, sus aptitudes técnicas y su sensibilidad en lo que han imaginado" (Eisner, 2004, p. 243).

Investigadores tales como Madura (1998), Fiske (1999), Deasy (2002), Catterall (2002), Eisner (2004), Andrzejczak, Trainin y Poldberg (2005), Sanders (2006), Senitt (2006), Kay (2008) y Rachlin (2008) sugieren los múltiples beneficios que las artes aportan al currículo de las escuelas. Andrzejczak, Trainin y Poldberg (2005) llevaron a cabo una investigación basada en una estrategia de enseñanza en la que utilizaron las artes visuales como parte de un proceso de pre-escritura. El mismo se desarrolló como parte de un proyecto de investigación acerca del efecto de las estrategias de las artes en el aprovechamiento académico de los estudiantes. Los autores citados, concluyeron que la incorporación de las artes visuales en el proceso de escritura trajo consigo el enriquecimiento en el lenguaje metafórico y sensorial del estudiante y, a su vez, la escritura añadió al trabajo artístico una nueva dimensión de la idea original.

Sanders (2006) llevó a cabo una investigación de enfoque fenomenológico, en la que participaron seis estudiantes de cuarto grado que estuvieron inmersos en un currículo de arte y escritura. A través de esta investigación, la autora exploró la manera en que se relacionan la composición artística con la composición escrita, y viceversa. Además, mediante el análisis de la experiencia del estudiante midió la efectividad de la enseñanza a través de modelos múltiples. Para esto, utilizó también la lectura mediante la clasificación por unidades temáticas, tales como poesía, historia, artistas, técnicas, entre otros.

En otra investigación, Rachlin (2008) identificó la necesidad de reforzar la destreza de escritura, ya que los estudiantes de escuela intermedia obtenían puntuaciones bajas en las pruebas estandarizadas y en la escuela superior sucedía lo mismo con las pruebas de cuarto año; en ambas, el problema era la presencia de errores al escribir. El investigador enfocó el diseño de un currículo, que denominó "Tengo algo que decir", en aquellos estudiantes cuya primera lengua no era el inglés. Éste se basó en el estudio de murales mexicanos para enseñar la escritura persuasiva. Se utilizó el arte como un organizador visual para la elaboración de sus ensayos. Las conclusiones fueron que, así como el arte es personal, la escritura también lo es. El autor, consideró que es mediante un enfoque personal que se desarrollan exitosamente ambas.

\subsection{El lenguaje, las artes visuales y el aprendizaje significativo}

La evolución de los medios masivos de comunicación, en particular Internet, permiten al ser humano acceder a todo tipo de información fácilmente. Ante esto, la escuela del siglo XXI tiene ante sí el reto de desarrollar estudiantes educados y sensibles, capaces de hacer uso y transferir la información a conocimientos que puedan ser utilizados en situaciones 
reales. De acuerdo con Álvarez (2006) el ser humano aprende de forma natural como respuesta a las experiencias que ha vivido a lo largo de los años. Es por eso, que no se debe desligar el aprendizaje de las emociones y sentimientos que surgen en el contexto social en que se desarrolló el nuevo conocimiento (Damasio, 2003). De esto se puede sugerir, que cuando la memoria del aprendizaje nuevo está asociada con una experiencia gratificante, el aprendizaje será perdurable (Dewey, 1934; Sousa, 2002).

Una idea común es que un trabajo artístico se identifique como algo aparte de la experiencia humana, no obstante, la experiencia ocurre en cada interacción que hace el ser humano con su ambiente (Dewey, 1934). La experiencia directa, por tanto, propicia conocimientos, siendo ésta una manera de adquirirlos. Según Sousa (2002) es el mecanismo que encauza la enseñanza a incorporar el nivel de significado atribuido al nuevo conocimiento. Entonces, si este aprendizaje se da en condiciones normales, será difícil de olvidar.

Las destrezas del lenguaje se desarrollan en el aprendiz desde el nacimiento. No obstante, éste añade los nuevos conocimientos a los que ya ha adquirido (Ausubel, 2002) para lograr llegar a conclusiones nuevas, aplicándolo, a su vez, a nuevas situaciones. De esta manera el niño que lee y escribe, cada vez más, cobrará confianza y seguridad en su propio aprendizaje.

\subsection{Marco conceptual y teórico}

El marco teórico de la investigación incorpora las definiciones y teorías de: Rabkin y Redmond (2004), para el constructo de artes visuales; Goodman (2003), para los constructos de lectura y escritura; y Ausubel (2002) para el constructo de aprendizaje significativo. A continuación, se presenta una descripción de las mismas de acuerdo con la relación de las variables investigadas.

\subsubsection{Artes Visuales}

Rabkin y Redmond (2004a) expusieron su perspectiva acerca de las artes visuales en la educación. La enseñanza y la integración curricular de las artes visuales, según los autores, son dos conceptos muy diferentes. Diferenciaron la integración como una estrategia eficaz para provocar cambios positivos en estudiantes, particularmente, aquéllos pertenecientes a la población por debajo del nivel de pobreza y en desventaja social y académica. Asimismo, los autores destacaron los beneficios cuando se incorporan, de manera estratégica e interdisciplinaria las artes visuales en otras materias. Entre éstos, identificaron la transferencia en el aprendizaje y, respecto a las artes visuales, el uso de la dimensión emocional, social y sensorial de éstas para convertirse en adultos exitosos, el aumento en el aprendizaje y la conexión del contenido con las experiencias personales y necesidades del estudiante para un mejor entendimiento del mundo.

\subsubsection{Lectura y escritura}

De acuerdo con Goodman (2003), el aprendizaje del lenguaje es un proceso mediante el cual se da la invención y la convención. El ser humano, según el autor, inventó el lenguaje para poder comunicarse, para aprender y para pensar; luego, al unirse cada individuo con sus invenciones personales y conformar la sociedad, se crean las convenciones sociales. De esta manera, las invenciones personales y convenciones sociales del lenguaje interactúan entre sí. 
El lenguaje escrito, según afirma Goodman (2003), es el resultado del uso del lenguaje oral. No obstante, de acuerdo con el autor, la lectura y la escritura son procesos que se desarrollan a la par ya que se construyen a partir de lo que se conoce; los niños observan lo impreso, responden a éste otorgándole significado, lo imitan y llegan al lenguaje escrito convencional.

\subsubsection{Aprendizaje significativo}

Ausubel (1962), propuso la teoría del aprendizaje significativo definiéndola como el proceso a través del cual una persona incorpora la nueva información de manera que ésta se relacione con la estructura cognitiva previamente existente. A este proceso lo denominó asimilación. Según Ausubel (1962, 2002), la estructura cognitiva previamente existente es el conjunto de conceptos e ideas que se encuentran en el aprendiz, así también como la organización de las mismas.

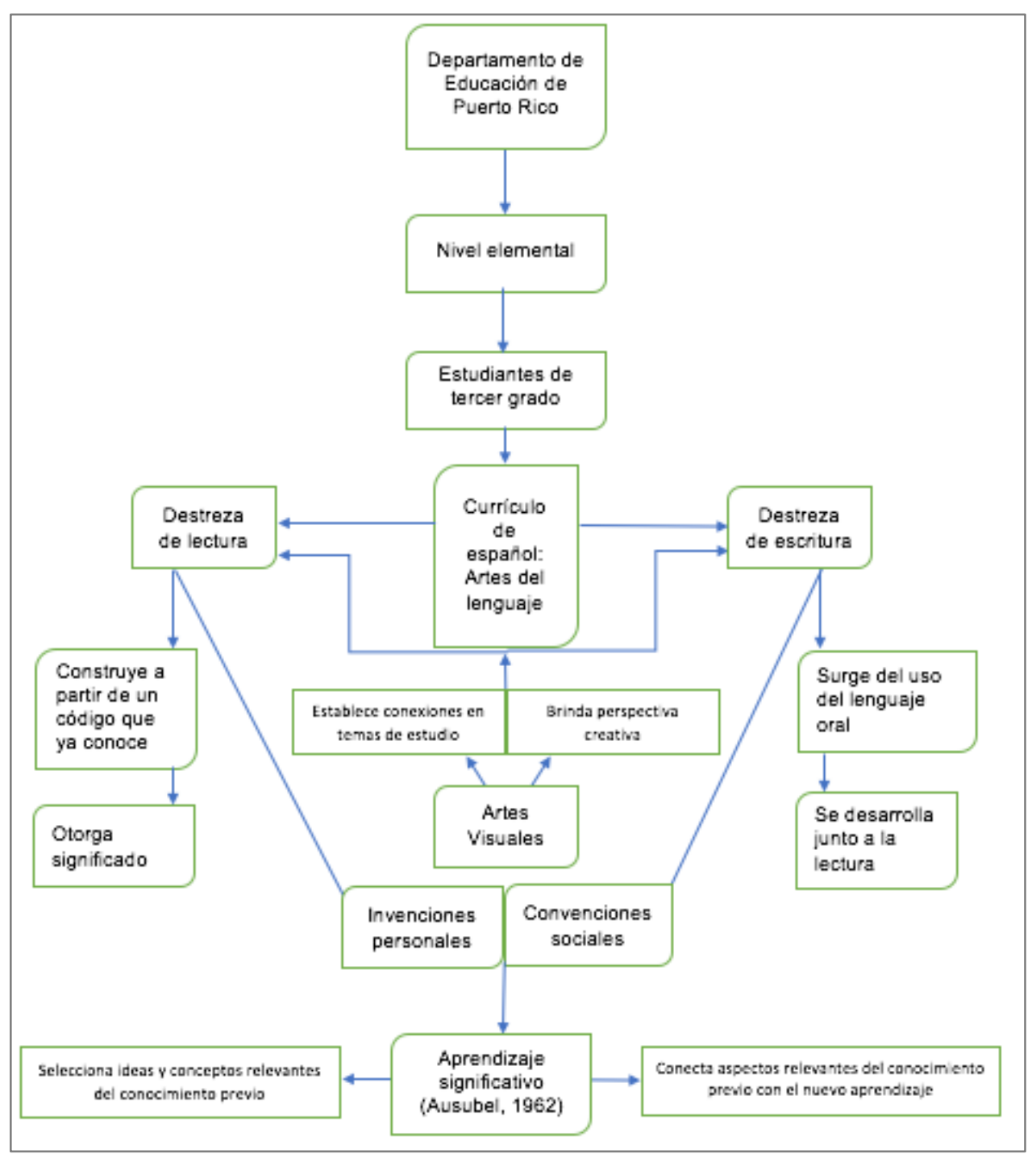

Figura 1. Marco conceptual teórico de la integración de las artes visuales en el currículo de español

Fuente: Elaboración propia. 
De acuerdo con el autor, para que se lleve a cabo este proceso: primero, debe tener motivación; segundo, debe poseer conocimientos previos; y, tercero, debe construir nuevos significados. Este proceso ocurre, según el autor, cuando el individuo selecciona las ideas y conceptos más relevantes y los añade al nuevo conocimiento, lo cual hace que la totalidad sea comprensible en cada experiencia de aprendizaje. No obstante, indican Ausubel (1962, 2002) y Ausubel, Novak y Hanesian (2009), que el aprendiz tiene que mostrar la capacidad de reconocer qué aspectos de lo que ya conoce son relevantes en la experiencia de aprendizaje actual.

\section{Métodos}

Para llevar a cabo esta investigación se utilizó un enfoque cuantitativo con un diseño cuasi-experimental. Creswell (2014) indica que el enfoque cuantitativo se debe utilizar, entre otras, en investigaciones para las que se requiera determinar el efecto en las variables de estudio luego de aplicar un tratamiento. Asimismo, Salkind (1999) sostiene que los diseños cuasi-experimentales permiten identificar las diferencias entre las variables, antes y después del tratamiento; a su vez, facilitan a los investigadores explorar preguntas que no podrían contestarse de otro modo. De acuerdo con McMillan y Schumacher (2010), el propósito de un diseño de investigación es proporcionar respuestas apropiadas a las preguntas de investigación planteadas.

Para ilustrar el mismo, el cuadro 4 muestra el diseño de investigación, en el que participó un grupo experimental y un grupo control. Los dos grupos fueron expuestos a cinco lecturas y a ejercicios de escritura durante un período de diez semanas.

Cuadro 4. Diseño de investigación

\begin{tabular}{lccc}
\hline \multicolumn{1}{c}{ GRUPOS } & PREPRUEBA & TRATAMIENTO & POSPRUEBA \\
\hline GE (Grupo experimental) & $\mathrm{O}_{1}$ & $\mathrm{X}$ & $\mathrm{O}_{2}$ \\
GC (Grupo control) & $\mathrm{O}_{1}$ & & $\mathrm{O}_{2}$ \\
\hline
\end{tabular}

$\mathrm{O}_{1}=$ preprueba, $\mathrm{O}_{2}=$ posprueba, $\mathrm{X}=$ tratamiento.

Fuente: Elaboración propia.

El grupo experimental (GE) leyó las historias y escribió mediante tareas en las que, para propósitos de esta investigación, se integró las artes visuales a través de un segmento de integración curricular diseñado para estos fines. El grupo control (GC) leyó las mismas historias y escribió mediante tareas en las que se llevó a cabo el desarrollo de estas destrezas de la manera tradicional, sin incorporar las artes visuales.

\subsection{Muestra}

La muestra de esta investigación fue de 21 estudiantes de tercer grado los cuales fueron seleccionados por conveniencia. Los dos grupos fueron heterogéneos, en los que se incluyó los estudiantes de la corriente regular y los estudiantes de educación especial de dos escuelas públicas rurales ubicadas en el suroeste de Puerto Rico.

\subsection{Instrumentos de investigación}

Los instrumentos de investigación fueron diseñados por la investigadora con la colaboración de una persona con vasta experiencia en la enseñanza y supervisión en el 
área académica de español. Para la elaboración de éstas, se consideraron los estándares de contenido y las expectativas para el tercer grado del programa de español del DEPR.

\subsubsection{Preprueba y posprueba de lectura}

La prueba de comprensión lectora contenía dos lecturas. Se incluyó 18 preguntas de respuestas múltiples, basadas en las lecturas. Cada pregunta constaba de tres alternativas como probable respuesta: una respuesta correcta y dos distractores. Se proveyó una hoja a cada estudiante para que marcara la respuesta correcta al ennegrecer la burbuja en la cual se encontraba la letra seleccionada. Mediante un estudio piloto, esta prueba obtuvo un coeficiente de confiabilidad alfa de Cronbach con valor de 0,82.

\subsubsection{Preprueba y posprueba de escritura}

La prueba de escritura creativa consistió en la presentación de dos imágenes de obras de arte, las que se solicitó al estudiante observar. A continuación, se pidió al mismo que basándose en lo que observó, escribiera un párrafo de no menos de ocho oraciones. Dicho párrafo se evaluó mediante el uso de la Rúbrica para evaluar la destreza de escritura, la cual contenía 13 criterios para cada una de las dos imágenes presentadas. Mediante un estudio piloto, esta prueba obtuvo un coeficiente de confiabilidad alfa de Cronbach con valor de 0,95 .

\subsubsection{Segmento de integración curricular}

Este segmento de integración curricular se basó en tareas de integración artística que fueron diseñadas para complementar las lecturas incluidas en el currículo de adquisición de la lengua el cual incluye la enseñanza del español como lenguaje materno integrado con la historia de Puerto Rico. Por consiguiente, para la construcción del mismo se tomó en consideración el contenido del Mapa curricular de Adquisición de la Lengua para tercer grado y la comunicación constante con la maestra de este grupo. De esta manera, durante diez semanas se incluyó actividades de los programas de español y de artes visuales apropiadas para su nivel.

\subsection{Procedimiento}

La investigación fue autorizada por el DEPR y la Junta de Revisión Institucional de la Pontificia Universidad Católica de Puerto Rico. Se explicó la investigación al director, maestros, estudiantes y padres participantes, de las escuelas seleccionadas. Se obtuvo el consentimiento y asentimiento informado de los participantes y tutores de éstos.

\section{Administración de preprueba de lectura y de escritura}

Una vez, obtenidos los documentos requeridos para realizar la investigación, se administró las prepruebas de lectura y de escritura, respectivamente, al grupo control (GC) y grupo experimental (GE).

\section{Administración de tratamiento a través de la integración de las artes visuales}

El tratamiento constó de la integración de las artes visuales a la enseñanza de la adquisición de la lengua de español por 30 minutos, al GE, durante diez semanas. Para dar dirección a esta etapa, la investigadora diseñó un segmento de integración curricular a través del cual tomó en consideración el Mapa curricular de Adquisición de la Lengua para tercer grado. Específicamente, la primera unidad temática es Soy Puerto Rico: 
autografías y narrativas personales a través de la cual los estudiantes estuvieron expuestos a actividades que los dirigieron a comprender que las biografías incluyen eventos y detalles de la vida de la persona. A medida que, la maestra de español los expuso a la lectura de autobiografías y a la identificación de aspectos tales como: la narrativa y el propósito del autor, la secuencia, los intereses, las capacidades y los valores personales, entre otros, la maestra de artes visuales le enseñó acerca de los autorretratos. Al mismo tiempo, identificaron similitudes entre éstos y las autobiografías. Además, establecieron la diferencia entre retrato y autorretrato de la misma manera que diferenciaron una biografía de una autobiografía. En otra actividad, con la maestra de español prepararon una línea del tiempo del pelotero Roberto Clemente, mientras que con la de artes visuales prepararon una línea del tiempo del pintor Francisco Oller, ambos personajes son considerados puertorriqueños famosos. Mientras leyeron Un pintor puertorriqueño sobresaliente: su vida y proyecciones (Cruz y Cruz-López, 1979) también observaron la obra del pintor José Campeche, el cual es el personaje principal de la misma. De igual manera, los estudiantes dibujaron, pintaron e hicieron grabados durante esta etapa de integración.

\section{Administración de posprueba de lectura y de escritura}

Al finalizar las diez semanas, los estudiantes de los dos grupos contestaron las pospruebas de lectura y de escritura, respectivamente, siendo los mismos instrumentos que fueron utilizados para la preprueba.

\section{Resultados y Conclusiones}

El análisis estadístico incluyó la prueba Mann-Whitney y la prueba t para grupos independientes, para analizar las puntuaciones de la preprueba y las puntuaciones de la posprueba, respectivamente. También se realizó la prueba Wilcoxon y la prueba t para muestras pareadas, para analizar las puntuaciones y determinar las diferencias entre los grupos. Para determinar los efectos del tratamiento en el GE se llevó a cabo el análisis de regresión lineal simple. Las hipótesis se sometieron a un nivel de significancia o alfa $(\boldsymbol{\alpha})$ de ,01 y ,05. Todos los análisis se realizaron a través del uso del programa estadístico SPSS Versión 24. A continuación, se presenta el cuadro 5 en la cual se incluye el promedio de las puntuaciones obtenidas por los estudiantes participantes de los dos grupos, para la posprueba de lectura y de escritura, respectivamente.

El cuadro 5 presenta los datos que se obtuvieron de la posprueba de lectura y de escritura. En la posprueba de lectura, las alternativas para responder a las preguntas, según el rango, son: 3 = totalmente correcta, $2=$ parcialmente correcta y $1=$ totalmente incorrecta. Esto implica que el promedio más alto para esta prueba es de 3. Mientras que, la posprueba de escritura fue evaluada mediante rúbrica cuyos criterios, según el rango, son: $4=$ capacitado, $3=$ se observan logros, $2=$ en desarrollo y $1=$ en inicio. El promedio máximo para esta prueba es de 4.

En el cuadro 5 se observa que, los estudiantes del GE obtuvieron puntuaciones más altas en la posprueba de lectura y de escritura al compararlas con las puntuaciones obtenidas por los estudiantes del GC. De acuerdo con estos datos, la prueba en la cual los estudiantes de ambos grupos tuvieron mejor ejecución fue la de lectura. No obstante, la prueba de escritura resultó una de mayor dificultad para ambos grupos, aunque el GC resultó con puntuaciones más bajas comparadas con las obtenidas por el GE. 
Cuadro 5. Datos obtenidos por los estudiantes participantes de los dos grupos investigados

\begin{tabular}{|c|c|c|c|}
\hline \multirow[b]{2}{*}{ PARTICIPANTE } & \multirow[b]{2}{*}{ *GRUPO } & \multicolumn{2}{|c|}{ POSPRUEBA } \\
\hline & & $\begin{array}{c}\text { Lectura } \\
\text { Valor máximo }=3,00 \\
\bar{x}\end{array}$ & $\begin{array}{c}\text { Escritura } \\
\text { Valor máximo }=4,00 \\
\bar{x}\end{array}$ \\
\hline 1 & GC & 2,44 & 1,12 \\
\hline 2 & GC & 2,06 & 2,31 \\
\hline 3 & GC & 2,33 & 2,08 \\
\hline 4 & GC & 1,89 & 1,04 \\
\hline 5 & GC & 1,89 & 1,38 \\
\hline 6 & GC & 2,22 & 1,08 \\
\hline 7 & GC & 2,11 & 1,50 \\
\hline 8 & GC & 2,11 & 1,00 \\
\hline 9 & GC & 2,33 & 1,46 \\
\hline 10 & GC & 2,67 & 2,00 \\
\hline 11 & GC & 2,06 & 1,27 \\
\hline 12 & GC & 2,33 & 1,38 \\
\hline 13 & GC & 2,39 & 1,35 \\
\hline 14 & GC & 2,22 & 1,77 \\
\hline 15 & GE & 2,67 & 2,35 \\
\hline 16 & GE & 2,72 & 2,19 \\
\hline 17 & GE & 2,72 & 3,65 \\
\hline 18 & GE & 2,72 & 1,96 \\
\hline 19 & GE & 2,50 & 2,04 \\
\hline 20 & GE & 2,78 & 2,04 \\
\hline 21 & GE & 2,56 & 2,04 \\
\hline 22 & GE & 2,83 & 1,96 \\
\hline 23 & GE & 2,56 & 2,88 \\
\hline 24 & GE & 2,78 & 2,50 \\
\hline 25 & GE & 2,83 & 2,46 \\
\hline 26 & GE & 2,89 & 2,27 \\
\hline 27 & GE & 2,67 & 2,92 \\
\hline 28 & GE & 2,94 & 3,50 \\
\hline 29 & GE & 2,61 & 2,08 \\
\hline 30 & GE & 2,72 & 2,73 \\
\hline 31 & GE & 2,83 & 2,42 \\
\hline
\end{tabular}

*Grupo: GC = grupo control; $\mathrm{GE}=$ grupo experimental; $\bar{x}=$ promedio.

Fuente: Elaboración propia.

\subsection{Diferencia entre las puntuaciones de la preprueba y de la posprueba de lectura, $\operatorname{del} G E$}

El resultado de la prueba Wilcoxon, para determinar si existía diferencia significativa entre las puntuaciones en la pre y la posprueba de lectura en el grupo experimental, resultó en un nivel de significancia de ,ooo, por lo que se encontró que existe diferencia significativa entre las medianas de las puntuaciones de la preprueba y posprueba de lectura. Además, la prueba $t$ pareada dio como resultado una diferencia de $\bar{x}=-, 54248$. El valor de $t=-9,190$ resultó con una significancia de ,000, por lo que se encontró diferencia significativa entre los promedios de las puntuaciones de la preprueba y posprueba de lectura en estudiantes del grupo experimental. La Figura 2 presenta la comparación de resultados entre pre y posprueba de lectura, del GE. 


\section{GRUPO EXPERIMENTAL}

Prueba de lectura

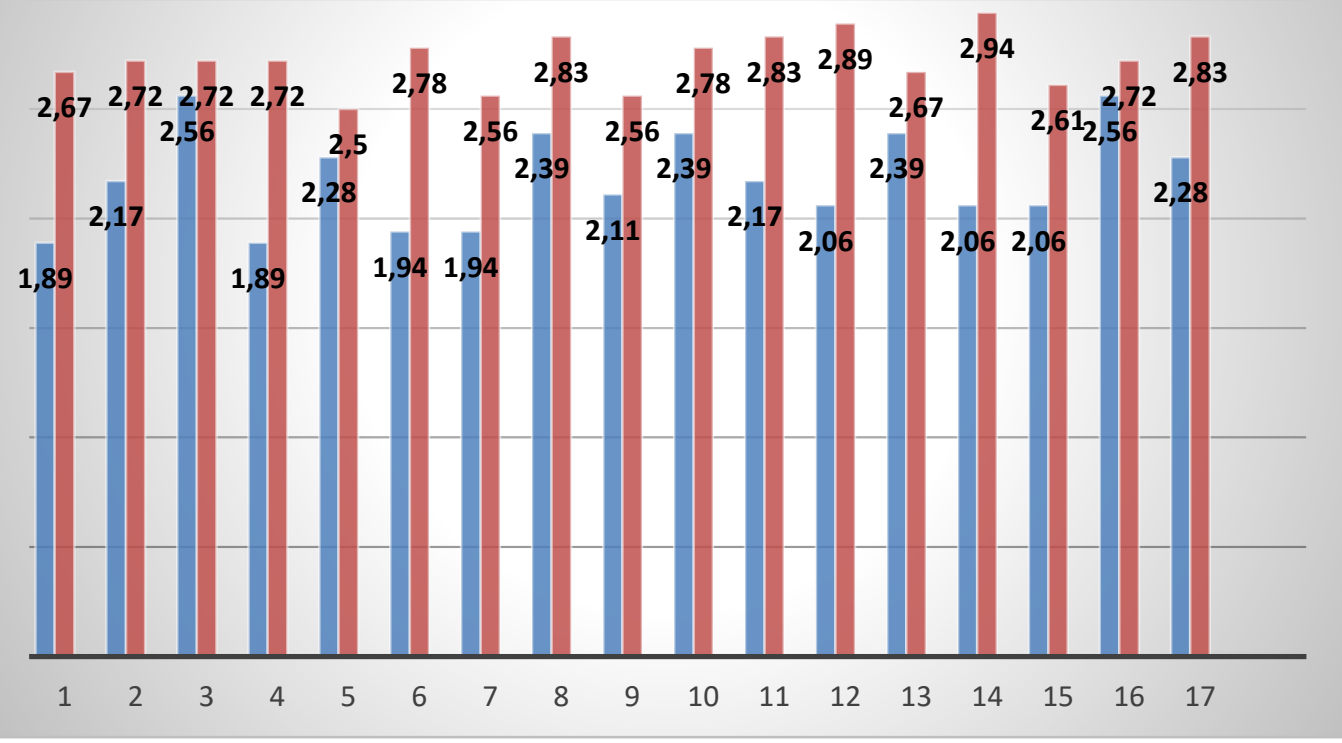

Preprueba $\square$ Posprueba

Figura 2. Comparación de resultados entre preprueba y posprueba de lectura, del GE Fuente: Elaboración propia.

Se observa en la figura 2 que todos los estudiantes del GE obtuvieron puntuaciones más altas en la posprueba de lectura al compararlas con las obtenidas en la preprueba.

\subsection{Diferencia entre las puntuaciones de la preprueba y de la posprueba de escritura, del $G E$}

El resultado de la prueba Wilcoxon, para determinar si existe diferencia significativa entre la pre y la posprueba de escritura en el grupo experimental, resultó en un nivel de significancia de ,000, por lo que se encontró que existe diferencia significativa entre las medianas de las puntuaciones de la preprueba y posprueba de escritura. Además, el resultado de la prueba $t$ pareada fue de $\bar{x}=-, 54248$. El valor de $t=-13,925$ resultó con una significancia de, 000 , por lo que se descubrió que existe diferencia significativa entre los promedios de las puntuaciones de la preprueba y posprueba de escritura en estudiantes del grupo experimental. La Figura 3 presenta la diferencia entre pre y pos. 


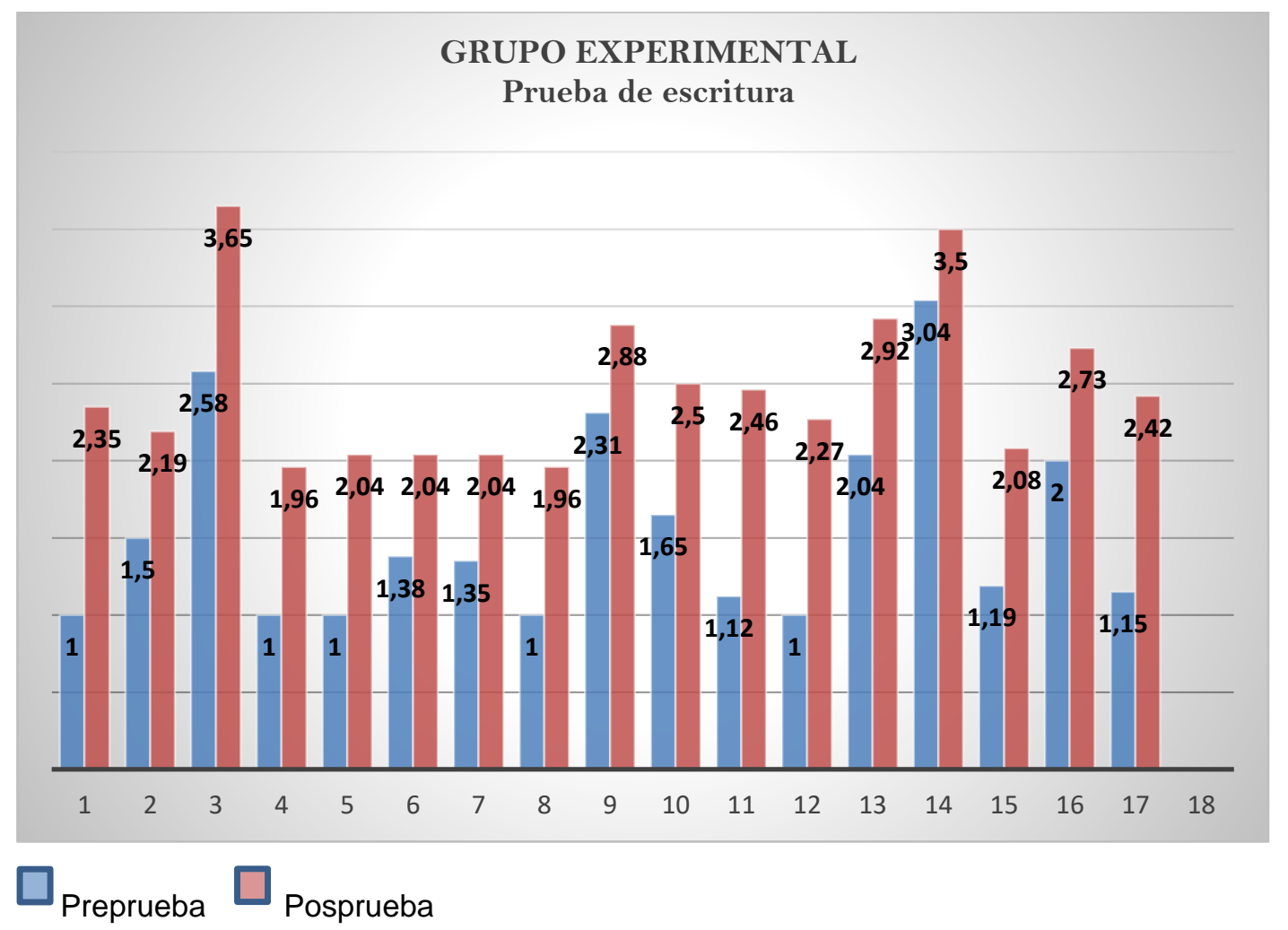

Figura 3. Comparación de resultados entre preprueba y posprueba de escritura, del GE Fuente: Elaboración propia.

Se observa en la figura 3 que todos los estudiantes del GE obtuvieron puntuaciones más altas en la posprueba de escritura al compararlas con las obtenidas en la preprueba.

\section{3. ¿Cuál es el efecto de la integración de las artes visuales al desarrollo de las destrezas de lectura en estudiantes de tercer grado de escuelas públicas del suroeste de Puerto Rico?}

Para contestar esta pregunta y la hipótesis planteada, se llevó a cabo el análisis de regresión lineal simple. Las magnitudes para medir la asociación se clasificaron mediante el uso de la tabla de correlación de Dancey y Reidy (2004). De acuerdo con los resultados, el valor $r=, 838$ y el valor $r$ square $=, 702$, lo cual indicó una correlación positiva fuerte $(0$ $\leq r \leq 1$ ), entre la preprueba de lectura y las puntuaciones de los estudiantes. Además, el coeficiente de regresión $B=$,059 indicó que, por cada punto adicional en la preprueba, la puntuación en la posprueba aumentará en ,059. En este caso, la variable independiente Preprueba de lectura (con un valor de $t=437$ ) tiene un nivel de significancia de 665 , mientras que, la variable independiente Grupo tiene un valor de $t$ positivo $(t=10,960)$ y significancia de ,000. La evidencia apunta que, no hay relación significativa entre las puntuaciones obtenidas por los estudiantes en la preprueba de lectura y en la posprueba de lectura, sin embargo, el estudiante que está en el grupo experimental tendrá mayor probabilidad de obtener puntuaciones más altas en la posprueba de lectura que el estudiante del grupo control, porque el coeficiente de la variable Grupo $(, 506)$ resultó estadísticamente significativo (significancia $=, 000$ ) y positivo. 


\section{4. ¿Cuál es el efecto de la integración de las artes visuales al desarrollo de las destrezas de escritura en estudiantes de tercer grado de escuelas públicas del suroeste de Puerto Rico?}

Para contestar esta pregunta y la hipótesis planteada, se llevó a cabo el análisis de regresión lineal simple. Las magnitudes para medir la asociación se clasificaron mediante el uso de la tabla de correlación de Dancey y Reidy (2004). De acuerdo con los resultados, el valor $r=, 958$ y el valor $r$ square $=, 917$, lo cual indica una correlación positiva fuerte $(0$ $\leq r \leq 1$ ) entre la preprueba de escritura y el grupo al que pertenecen los estudiantes con sus puntuaciones en la posprueba. Además, el coeficiente de regresión $B=, 815$ indica que, por cada punto adicional en la preprueba, la puntuación en la posprueba aumentará en ,815. En este caso, las variables independientes Preprueba de Escritura $(t=11,341)$ y Grupo $(t=10,960)$ son estadísticamente significativas $(, 000<, 05)$ y positivas. Hay evidencia estadística de que a mayor puntuación en la preprueba de escritura mayor será la puntuación en la posprueba de escritura. El estudiante que está en el grupo experimental tendrá una mayor probabilidad de obtener más puntuación en la posprueba de escritura que el estudiante del grupo control, porque el coeficiente de la variable Grupo $(, 819)$ resultó estadísticamente significativo (significancia $=, 000$ ), y positivo.

\section{Discusión y resultados}

El propósito de esta investigación fue determinar el efecto de la integración de las artes visuales en el desarrollo de las destrezas de lectura y escritura en estudiantes de tercer grado en escuelas públicas rurales del suroeste de Puerto Rico. Para la discusión de los resultados, se tomó en consideración la perspectiva de las artes visuales en la educación de Rabkin y Redmond (2004), la teoría de la invención y convención del lenguaje de Goodman (2003) y la teoría del aprendizaje significativo de Ausubel (1962).

El hallazgo de mejoramiento en la lectura coincide con lo que encontró Wilhelm (2002). Este autor realizó una investigación con enfoque cualitativo, a través de un estudio etnográfico cuya duración fue de nueve semanas. En la misma se aplicó el uso de métodos de entrenamiento visual a través de las artes visuales. Los dos estudiantes que participaron en la referida investigación evidenciaron aumento en las destrezas de lectura relacionadas con rapidez, comprensión y aportaciones personales, respecto a la lectura. El hallazgo de esta investigación al cual se hace referencia coincide, además, con los resultados de Rabkin y Redmond (2004b) quienes reportaron que los estudiantes participantes de escuelas cuyo currículo incluía programas de integración de artes visuales en la clase de español, entre otras materias, mejoraron las puntuaciones en las pruebas estandarizadas de lectura más rápidamente que los estudiantes de escuelas que no participaron de dicha integración curricular. Cónsono con los resultados de esta investigación, se encuentran los hallazgos de López-Carrasquillo (2010) quien llevó a cabo un proyecto de alfabetización con adultos que no sabían leer. El investigador reportó que los participantes lograron aprender a leer mediante literatura cuyos temas le interesaban y que eran pertinentes para ellos. Según el autor, este factor contribuyó al desarrollo de la motivación y un mayor compromiso del estudiante con su propio aprendizaje.

Por otro lado, los resultados de incremento en las puntuaciones de la prueba de escritura entre pre y pos, en estudiantes del grupo experimental, coinciden con los resultados que 
reportaron Madura (1998), Andrzejczak et al. (2005) y Connel-Ross (2006). Los autores identificaron resultados favorables en la escritura, al concluir sus respectivas investigaciones de enfoque cualitativo, a través de las cuales integraron a la escritura aspectos de las artes visuales tales como la utilización y producción de imágenes artísticas y el aspecto estético de la obra. Entre los logros, informaron que la integración de las artes visuales ayudó a los estudiantes a: enfocarse en la tarea, escribir los detalles, desarrollar lenguaje metafórico y sensorial, describir, evaluar e interpretar el tema mediante dibujos y escritos. Así también contribuyó a la motivación de estudiantes reacios a involucrarse en la escritura. Además, el hallazgo al cual se hace referencia coincide con los resultados que presentó Fiske (1999). El autor determinó que los estudiantes que estuvieron expuestos a la educación artística mostraron cambios en el proceso de aprender, tales como, el desarrollo del aprendizaje por descubrimiento y la iniciativa de aprender.

Efecto de la integración de las artes visuales en lectura. El efecto positivo del tratamiento en la lectura coincide con los resultados que reportó Catteral (2002) los cuales indican que el entrenamiento visual ayudó a desarrollar la interpretación de textos de los estudiantes que participaron de la integración de las artes visuales. Este resultado es cónsono, además, con Weissman (2004) quien encontró que los estudiantes expuestos a esta variable en una escuela cuyos estudiantes no lograban el desempeño esperado en las pruebas estandarizadas, triplicaron las puntuaciones en el área de comprensión lectora, en el término de cinco años. No obstante, los resultados son contrarios a los de Burger y Winner (2000) ya que los autores no encontraron asociación entre la enseñanza de las artes visuales y el mejoramiento en la lectura.

Efecto de la integración de las artes visuales en la escritura. Mediante el análisis de los datos, se determinó que la integración de las artes visuales tuvo un efecto positivo y estadísticamente significativo, tanto en el desarrollo de las destrezas de lectura como en el de las destrezas de escritura. En ambos casos, se encontró que los estudiantes expuestos a la variable artes visuales mostraron un incremento en las puntuaciones obtenidas en las pospruebas con relación a las que habían obtenido en las prepruebas.

Este hallazgo, coincide con el de Deasy (2000), Connel- Ross (2006), Sanders (2006) y Leigh (2007) quienes reportaron que los estudiantes que han estado expuestos a la integración de las artes visuales experimentaron incremento en el aprovechamiento académico, particularmente, en las destrezas de escritura. Además, Eisner (2004) indica que el uso de estrategias de enseñanza que apelan a la motivación y al interés del estudiante tiene como resultado que los aprendices logren desarrollar en mayor escala sus capacidades, comparados con los logros de los estudiantes cuya enseñanza se le ofrece del modo tradicional. Por otra parte, los hallazgos difieren de Craffey (2009) quien luego de realizar una investigación cuasi experimental para determinar la probable relación entre el uso de diversas modalidades artísticas y el desarrollo la adquisición del lenguaje, concluyó que no hubo conexiones significativas entre el uso de las modalidades artísticas y la adquisición del lenguaje.

Efecto de la integración de las artes visuales en la lectura y la escritura. El efecto positivo de la integración de las artes visuales tanto en la lectura como en la escritura coincide con la teoría de aprendizaje significativo de Ausubel (1962) quien expuso que este aprendizaje ocurre cuando el individuo incorpora la nueva información de manera que ésta se relacione con la estructura cognitiva previamente existente. Para que esto ocurra, según el autor, el 
lector o escritor debe estar motivado, poseer conocimientos previos y construir nuevos significados. En este caso, los estudiantes del grupo experimental estuvieron expuestos a la enseñanza del español como su lengua materna, mientras participaban de manera integrada de actividades artísticas que conectaban con el conocimiento adquirido. Asimismo, en cada experiencia de aprendizaje, el estudiante como lector y escritor seleccionó los conceptos más relevantes y los añadió al nuevo conocimiento lo que hizo del mismo uno más comprensible. De acuerdo con los resultados de la investigación, se infiere que la integración de las artes visuales fue la estrategia que aportó la motivación para lograr los siguientes pasos hacia el desarrollo de las destrezas de lectura y escritura en los estudiantes de tercer grado, del grupo experimental.

En conclusión, la integración de las artes visuales en el desarrollo de las destrezas de lectura y escritura tuvo un efecto positivo en el rendimiento de los estudiantes expuestos a la misma, pues mostraron mejor rendimiento en las pospruebas de lectura y escritura que los estudiantes que no tuvieron esta intervención. No obstante, es importante señalar que estos resultados se limitan a los estudiantes de tercer grado participantes de la investigación, por lo que no son generalizables a la población de estudiantes de tercer grado. Se sugiere para futuras investigaciones que la muestra sea seleccionada aleatoriamente y que se aplique el tratamiento por un período mayor de tiempo.

\section{Referencias}

Álvarez-Pérez, H. (2006). Los hallazgos de las neurociencias y su aplicabilidad a la sala de clases: Teoría y práctica. Guaynabo: Ediciones Santillana.

Andrzejczak, N., Trainin, G. y Poldberg, M. (2005). From image to text: Using images in the writing process. International Journal of Education \&' the Arts, 6(12).

Ausubel, D. (1962, November). Learning by discovery. Educational Leadership, 2O(2), 13-117.

Ausubel, D. (2002). Adquisición y retención del conocimiento: Una perspectiva cognitiva. Barcelona: Paidós.

Ausubel, D., Novak, J. y Hanesian, H. (2009). Psicología educativa: Un punto de vista cognoscitivo. Ciudad de México: Trillas.

Burger, K. y Winner, E. (Fall 2000). Instruction in visual art: Can it help children learn to read? Journal of Aesthetic Education, 34(3-4), 277-293.

Catterall, J. (2002). The arts and the transfer of learning. En R. Deasy (Ed.), Critical links: Learning in the arts and student academic and social development (pp. 151-157). Washington, DC: Arts Education Partnership.

Coady, K. (2007). No writer left behind: Examining the reading-writing connection in the reading first classroom through a teacher study group. Tesis doctoral. Universidad del Estado de Georgia, Atlanta, GA.

Connel-Ross, B. (2006). Ekphrasis: Putting the art into language arts drawing on art and visual representation to teach writing. Tesis doctoral, Universidad de Texas, El Paso, TX.

Craffey, J. (2009). An evaluation of how the arts facilitate language acquisition through multisensory modalities. Tesis doctoral. Universidad de Northcentral, San Diego, CA.

Creswell, J. (2014). Research design: Qualitative, quantitative, and mixed methods approaches. Los Angeles, CA: Sage Publications. 
Cruz, E. y Cruz-López, D. (1979). Un pintor puertorriqueño sobresaliente: Su vida y proyecciones. En A. Pastor, R. Guzmán y K. Hester (Eds.), Páginas de ayer y de hoy: Por el mundo del cuento y la aventura, libro VI (pp. 2016-214). River Forest, IL: Laidlaw Brothers.

Damasio, A. (2003). Looking for Spinoza: Joy, sorrow, and the feeling brain. Nueva York, NY: Harvest Book Hardcourt.

Dancey, C. y Reidy, J. (2004). Statistics without maths for psychology: Using SPSS for Windows. Londres: Prentice Hall.

Deasy, R. (2002). Critical links: Learning in the arts and student academic and social development. Washington, DC: Arts Education Partnership.

Departamento de Educación de Puerto Rico. (2013). Carta circular núm. 5 - 2013 - 2014. Política pública sobre la organización y la oferta curricular del programa de bellas artes en los niveles elemental y secundario de las escuelas públicas de Puerto Rico. San Juan: Autor.

Departamento de Educación de Puerto Rico. (2014a). Carta circular núm. 5 - 2014 - 2015. Política pública sobre la clasificación de las escuelas e implementación de estrategias de transformación para mejorar el aprovechamiento académico de los estudiantes de acuerdo con el plan de flexibilidad. San Juan: Autor.

Departamento de Educación de Puerto Rico. (2015a). Carta circular núm. 4-2015-2016. Política pública sobre la organización y la oferta curricular del programa de español como lengua materna en los niveles primario y secundario de las escuelas públicas de Puerto Rico. San Juan: Autor.

Departamento de Educación de Puerto Rico. (2014b). Estándares de contenido y expectativas de grado de Puerto Rico: Programa de español. San Juan: Autor.

Departamento de Educación de Puerto Rico. (2015b). Informe del perfil escolar. San Juan: Autor.

Departamento de Educación de Puerto Rico. (2003a). Marco curricular: Programa de bellas bellas artes. San Juan: Instituto Nacional para el Desarrollo Curricular.

Departamento de Educación de Puerto Rico. (2003b). Marco curricular: Programa de español. San Juan: Instituto Nacional para el Desarrollo Curricular.

Dewey, J. (1934). Art as experience. Nueva York, NY: Perigree Books.

Dollins, C. (2001). The connection between reading and writing for early readers. Tesis doctoral, Universidad de Pepperdine, Malibu, CA.

Eisner, E. (1972). Educar la visión artística. Barcelona: Paidós Ibérica.

Eisner, E. (2004). El arte y la creación de la mente. Barcelona: Paidós Ibérica.

Fiske, E. (1999). Champions of change: The impact of the arts of learning. Washington, DC: Arts Education Partnership.

Fons, M. (2010). Leer y escribir: 10 ideas claves para los primeros pasos. Con firma 2010: Leer para aprender. Leer en la era digital. Recuperado de https://sede.educacion.gob.es/publiventa/con-firma-2010-leer-para-aprender-leer-en-laera-digital/educacion/13939. https://doi.org/10.4438/978/-84-369-4970-4

Freire, P. (2004). Cartas a quien pretende enseñar. Buenos Aires: Siglo XXI.

Goodman, K. (2003). El aprendizaje y la enseñanza de la lectura y la escritura. Enunciación, 8(1), 77-78. 
Goodman, K. (2002). El proceso de lectura: Consideraciones a través de las lenguas y del desarrollo. En E. Ferreiro y M. Palacios, M. (Eds.), Nuevas perspectivas sobre los procesos de lectura y escritura (pp. 13-28). Coyoacán: Siglo Veintiuno Editores.

Goodman, K. (1989). Lenguaje integral. Mérida: Editorial Venezolana.

Goodman, K. (1995). Lenguaje integral: Un camino fácil para el desarrollo del lenguaje. Textos de didáctica de la lengua y la literatura, 3(3), 77-91.

Goodman, Y. y Altwerger, B. (1981). A study of the development of literacy in preschool children. Ocassional Papers, Program in Language of Literacy. Tucson, AZ: University of Arizona.

Graves, D. (1973). Children's writing: Research directions and hypotheses based upon an examination of the writing processes of seven years old children. Tesis doctoral. Unversidad de Nueva York, Buffalo, NY.

Hartse, J., Burke, C. y Woodward, V. (1982). Children's language and world: Initial encounters with print. En J. Langer y M. Smith-Burke (Eds.), Bridging the gap: Reader meets author (pp. 105-131). Newark, DE: International Reading Association.

Leigh, S. (2007). Drawing, writing, and second graders. Tesis doctoral. Universidad del Sur de California, Columbia, SC.

Kay, L. (2008). Art education pedagogy and practice with adolescent students at -risk in alternative schools. Tesis doctoral, Universidad del Norte de Illinois, DeKalb,IL.

Kontos, S. y Huba, N. (1983, junio). The development and function of print awareness. Comunicación presentada en el Biennal Meeting of the Society for Research in Child Development. ERIC Document Reproduction Service No. ED 230-237.

Krishna, P. (2004). La educación correcta para el siglo XXI. Recuperado de http://www.pkrishna.org/spanish/right_education_spanish.html

López-Carrasquillo, M. (2010). Marginados en un mundo de letras: 14 principios para enseñar con amor, compasión y alegría. San Juan: Reliable Graphics.

Madura, S. (1998). Transitional readers and writers respond to literature through discussion, writing and art. Tesis doctoral, Universidad de Nevada, Reno, NV.

Maeso-Rubio, F. (2003). El arte de construir el conocimiento artístico. El diseño curricular de educación artística en la escuela primaria. En R. Marín Viadel (Ed.), Didáctica de la educación artística (pp. 229-271). Madrid: Pearson Educación.

Mass, L. (1982). Developing concepts of literacy in young children. The Reading Teacher, 35(6), 670-675.

McMillan, J. y Schumacher, S. (2010). Research in education: Evidence-based inquiry. Boston, MA: Pearson Education.

Public Law 89-10. (1965, April). Elementary and Secondary Education Act. Washington, DC: United State Congress.

Public Law 107-1 10. (2002, January). An act to close the achievement gap with accountability, flexibility, and choice, so that no child left behind. Washington, DC: 107th United State Congress.

Rabkin, N. y Redmond, R. (2004a). Learning and the arts. En N. Rabkin y R. Redmond (Eds.), Putting the arts in the picture: Refraiming education in the 21 st century (pp. 5-15). Chicago, IL: Columbia College Chicago. 
Rabkin, N. y Redmond, R. (2004b). Putting the arts in the picture: Refraiming education in the in the 21 st century. En N. Rabkin y R. Redmond (Eds.), Putting the arts in the picture: Refraiming education in the 21 st century (pp. 127-157). Chicago, IL: Columbia College Chicago.

Rabkin, N. y Redmond, R. (2006). The arts make a difference. Educational Leadership, 63(5), 60-64.

Rachlin, C. (2008). I have something to say: Using art to teach the writing of persuasive essays. Tesis de Maestría. Universidad de California, San Diego, CA.

Reyes-Tosta, M. (2013). El aprendizaje del español y del inglés en Puerto Rico y sus influencias extranjeras. Scientific International Journal, 10(3), 44-53.

Rolón-Collazo, L. (2013). Leer para escribir y escribir para leer: Propuesta sobre el proceso de escritura, el entorno como texto y la complicidad entre lectura y escritura. El Sol: Revista de la Asociación de Maestros de Puerto Rico, 54(2), 32-34.

Ruiz, D. (1998). La alfabetización temprana en el ambiente preescolar: Nuevas perspectivas para aprender a leer y escribir. Río Piedras, PR: Departamento de Programas y Enseñanza, Universidad de Puerto Rico.

Salkind, N. (1999). Métodos de investigación. Ciudad de México: Prentice Hall.

Sanders, J. (2006). Qualitative study of students' composing experiences in art-infused writing curriculum. Tesis doctoral. Universidad de Florida, Gainesville, FL.

Santiago-Villafañe, R. y Figarella-García, F. (2012). Más allá de las disciplinas: Competencia para la vida. El Sol: Revista de la Asociación de Maestros de Puerto Rico, 53(3), 22-26.

Senitt, A. (2006). Signs of substance: An investigation regarding the confluence of art and language in an elementary school context. Tesis doctoral. Universidad Concordia, Canadá.

Schneuwly, B. (2010). ¿Qué es escribir? Herramientas y aprendizaje de la escritura. Con firma 2010: Leer para aprender. Leer en la era digital. Madrid: Ministerio de Educación. https://doi.org/10.4438/978/-84-369-4970-4

Solé, I. (2010). Ocho preguntas en torno a la lectura y ocho respuestas no tan evidentes. Con firma 2010: Leer para aprender. Leer en la era digital. Madrid: Ministerio de Educación. https://doi.org/10.4438/978/-84-369-4970-4

Sousa, D. (2002). Como aprende el cerebro: Una guía para el maestro en la clase. Thousands Oaks, CA: Corwin Press.

United States Department of Education. (2015). Puerto Rico Elementary \& Secondary Education Act Flexibility Request: Approved. Washington, DC: Author.

Vygotsky, L. (1995). Pensamiento y lenguaje. Buenos Aires: Ediciones Fausto.

Weissman, D. (2004). You can't get much better than that. En N. Rabkin y R. Redmond (Eds.), Putting the arts in the picture: Refraiming education in the 21st century (pp. 17-48). Chicago, IL: Columbia College Chicago.

Wilhelm, J. (2000). Reading is seeing: Using visual response to improve the literary Reading of recluctant readers. En R. Deasy (Ed.), Critical links: Learning in the arts and student academic and social development (pp. 48-72). Washington, DC: Arts Education Partnership. 


\section{Breve CV de los autores}

\section{Wanda I. Padilla Martínez}

Profesora de Artes Visuales del Departamento de Educación de Puerto Rico. Ha coordinado proyectos para estudiantes, dirigidos a fortalecer las destrezas académicas básicas; entre éstos: Ayúdame a estudiar, Exploradores del ambiente y Pequeños Periodistas. Además, perteneció a las organizaciones Academia de Mentores de Puerto Rico, Coaching for Change y Living with a Star: Starpartners, Capítulo de Puerto Rico, NASA, entre otras. Doctora en Educación: Currículo y Enseñanza de la Pontificia Universidad Católica de Puerto Rico, Recinto de Ponce. Maestría en Artes Plásticas: Grabado, de la Universidad Interamericana de Puerto Rico, Recinto de San Germán y Bachillerato en Educación Secundaria en Arte de la Universidad de Puerto Rico, Recinto de Río Piedras. ORCID ID: 0000-0002-2696-2983. Email: padilla.wanda@gmail.com

\section{Jaime Ortiz-Vega}

Catedrático y Director de la Escuela Graduada en Educación de la Pontificia Universidad Católica de Puerto Rico. Doctor en Educación: Gerencia y Liderazgo Educativo de New York University. Consultor para agencias gubernamentales y privadas en áreas de planificación estratégica, gerencia educativa, currículo y enseñanza. Miembro de la American Educational Research Association y de Association for Supervision and Curriculum Development, International Level.Ex presidente del Capítulo de Puerto Rico de Asociación for Supervision and Curriculum Development. Poseedor de varias publicaciones en revistas académicas. ORCID ID: 0000-0003-0310-9362. Email: jortiz@pucpr.edu 